\title{
Neoadjuvant chemotherapy with S-1 for scirrhous gastric cancer: a pilot study
}

\author{
Taira Kinoshita ${ }^{1}$, Masaru Konishi ${ }^{1}$, Toshio Nakagohri ${ }^{1}$, Kazuo Inoue ${ }^{1}$, Tatsuya Oda ${ }^{1}$, \\ Shinichiro Takahashi ${ }^{1}$, Narikazu Boku ${ }^{2}$, Atsushi $\mathrm{Ohtsu}^{2}$, and Shigeaki Yoshida ${ }^{2}$ \\ ${ }^{1}$ Department of Surgical Oncology, National Cancer Center Hospital East, 6-5-1 Kashiwanoha, Kashiwa, Chiba 277-8577, Japan \\ ${ }^{2}$ Department of Medical Oncology, National Cancer Center Hospital East, Kashiwa, Chiba, Japan
}

\begin{abstract}
We conducted a pilot study using S-1 (TS-1 $\left.{ }^{\circledR}\right)$, a novel oral derivative of 5-fluorouracil, as neoadjuvant chemotherapy for potentially resectable scirrhous gastric cancer. The neoadjuvant chemotherapy consisted of two courses (each, 4-week administration and 2-week withdrawal) of S-1 at $100-120 \mathrm{mg} /$ body per day. Five patients were enrolled in this pilot study and underwent resection. The response rate for the neoadjuvant chemotherapy was $60 \%$ (three partial response [PR]; two stable disease [SD]). Three of the five patients received curative resection; the other two patients received noncurative resection because of localized peritoneal dissemination and positive results on cytological examination of the abdominal washing. No toxicity of grade 3 or more was exhibited during the two courses of chemotherapy. Pathological examination of the resected specimens revealed a marked reduction in the distribution of viable cancer cells in the stomach in the three patients with PR. In one of these patients, pathological findings suggestive of the possibility of disappearance of the cancer cells in the perigastric and paraaortic lymph nodes were noted. Because of the unexpectedly high response to $S-1$, we consider that the efficacy of $S-1$ as neoadjuvant chemotherapy for scirrhous gastric cancer should be verified by phase II and III trials.
\end{abstract}

Key words Scirrhous gastric cancer · S-1 · Neoadjuvant chemotherapy

\section{Introduction}

Scirrhous gastric cancer, also designated as linitis plastica or Borrmann type 4 tumor, is known for its aggressive behavior and poor prognosis. Even with recent developments in early detection and new anticancer drugs, treatment results for scirrhous gastric cancer

Offprint requests to: T. Kinoshita

Received: October 4, 2002 / Accepted: January 27, 2003 remain almost unchanged. Causes of the poor prognosis are difficulties in early discovery and resistance to all intensive chemotherapeutic agents. Extended surgical procedures, such as left upper abdominal evisceration, have a limited effect, and only at a certain stage [1]. Because of the frustration of being unable to improve survival for scirrhous gastric cancer, we conducted a phase II trial of sequential high-dose methotrexate and fluorouracil combined with doxorubicin (FAMTX) as neoadjuvant chemotherapy for potentially resectable scirrhous gastric cancer [2]. FAMTX was feasible and showed a high resectability rate, but no improvement in 2-year survival was obtained and the toxicity was moderate; therefore, we stopped the phase II trial at 20 cases.

$\mathrm{S}-1$ is a dihydropyrimidine dehydrogenase (DPD)inhibitory fluoropyrimidine (DIF) which showed the highest response rate among many oral anticancer agents against unresectable advanced gastric cancer in early and late phase II studies [3-5]. In these phase II trials, $\mathrm{S}-1$ showed a $33 \%$ response rate against scirrhous gastric cancer. Low toxicity of S-1 in the phase II trial was also one reason why we decided to start neoadjuvant chemotherapy using S-1 for scirrhous gastric cancer.

\section{Patients and methods}

Five patients were enrolled in this pilot study. All patients were diagnosed as having typical scirrhous gastric cancer by imaging diagnosis, with no incurable findings, including ascites, liver metastasis, shrinkage of the colon, and severe lymph node metastases, findings which were also confirmed by laparoscopic examination under general anesthesia. After confirmation of the resectable stage of scirrhous cancer, S- 1 was administered at 50 or $60 \mathrm{mg} /$ body $\times 2$ per day. One course of medication comprised 4-week administration and 2-week withdrawal. 
After two courses of S-1 administration, patients underwent resection. The toxicity of and response to this neoadjuvant chemotherapy were evaluated according to the National Cancer Institute Common Toxicity Criteria (NCI-CTC) and the Japanese classification of gastric carcinoma [6], respectively. The response of primary foci was evaluated by the criteria of the Japanese Research Society of Gastric Cancer for "c-lesions." Complete response (CR) was defined as the disappearance of all invasive findings, and partial response (PR) as more than a $50 \%$ decrease of the affected area on a barium-filled $\mathrm{X}$-ray film taken in the same position as that before treatment. Progressive disease (PD) was defined as an increase in lesions by $25 \%$, or the appearance of new lesions. The remaining cases were classified as stable disease (SD). Resectability rate and histological effects in the resected specimens were also evaluated.

\section{Results}

None of the five patients had any visible peritoneal dissemination, but two patients showed positive results on cytological examination of the abdominal washing from the Douglas cavity under laparoscopic examination. No toxicity of grade 3 or more was observed. The only toxicity was grade 2 pigmentation changes, in two patients.

All patients finished two courses of chemotherapy on schedule and the response rate of the neoadjuvant chemotherapy was $60 \%$ (three PR; two SD) according to the Japanese classification of gastric carcinoma [6]. Table 1 shows the laparoscopic findings, response to neoadjuvant chemotherapy, and operative and pathological findings of each patient according to the Japanese classification of gastric carcinoma. Two patients with positive results on cytological examina-tion also showed positive cytological results at laparotomy after chemotherapy. One of the two patients had localized peritoneal dissemination in the lesser omentum bursa which seemed impossible to detect at initial laparoscopy. All patients received total gastrectomy with splenectomy and D2 lymph node dissection, as well as extensive sampling of the paraaortic lymph nodes. Resection was curative in three patients according to the Japanese classification of gastric carcinoma. No serious complications developed after resection. Two patients showed mild pancreatorrhea, which prolonged their hospital stay for 2-3 weeks.

Figures 1 and 2 show fluoroscopic examination results in cases 3 and 4, respectively, before and after preoperative chemotherapy.

Table 1. Summary of findings in patients in the pilot study

\begin{tabular}{lcccc}
\hline $\begin{array}{l}\text { Case } \\
\text { No. }\end{array}$ & $\begin{array}{c}\text { Laparoscopic } \\
\text { findings }\end{array}$ & $\begin{array}{c}\text { Response to } \\
\text { chemotherapy }\end{array}$ & $\begin{array}{c}\text { Operative } \\
\text { findings }\end{array}$ & $\begin{array}{c}\text { Pathological } \\
\text { findings }\end{array}$ \\
\hline 1 & H0,P0,CY0,T3,Nx & SD & H0,P0,CY0,T3,N0 & pT3,pN0 \\
2 & H0,P0,CY0,T3,Nx & PR & H0,P0,CY0,T3,N0 & pT2,pN0 \\
3 & H0,P0,CY1,T3,Nx & PR & H0,P0,CY1,T2,N2 & pT2,pN3 \\
4 & H0,P0,CY0,T3,Nx & PR & H0.P0,CY0,T2,N2 & pT2,pN0 \\
5 & H0,P0,CY1,T3,Nx & SD & H0,P1,CY1,T3,N2 & pT3,pN2 \\
\hline
\end{tabular}

H0, No hepatic metastasis; P0, no peritoneal dissemination; $x$, not evaluated; CY0, negative result on cytological examination of the abdominal washing; CY1, positive result on cytological examination of the abdominal washing; SD, stable disease; PR, partial response
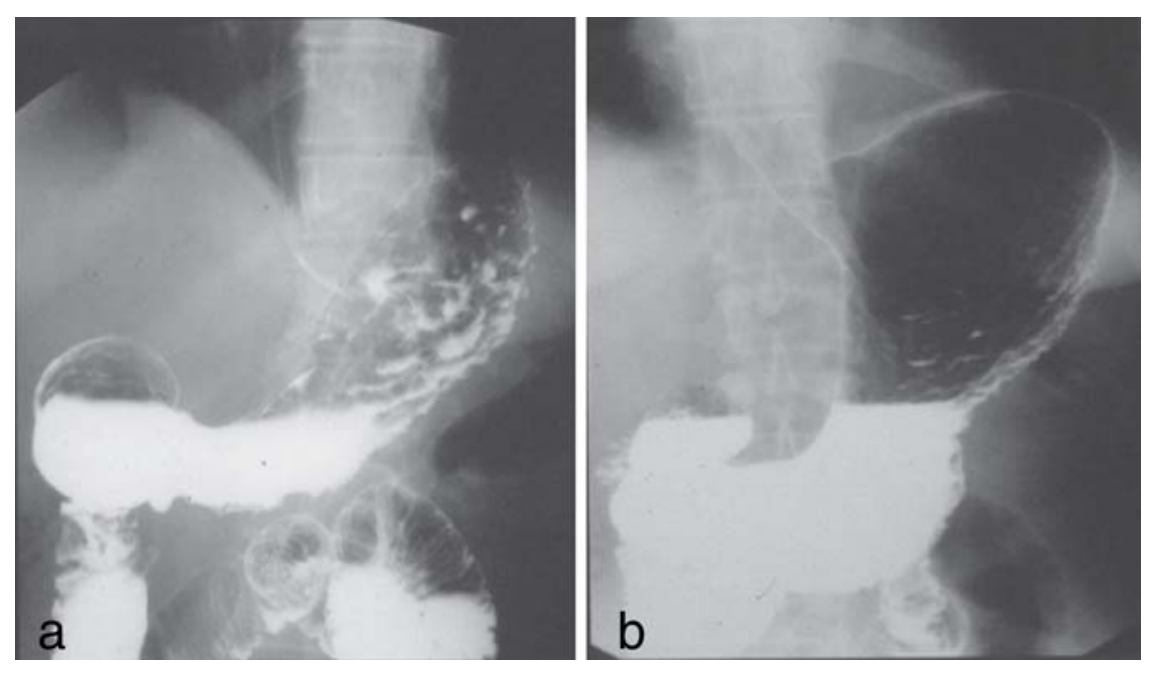

Fig. 1a,b. Fluoroscopic examination of case 3. a Before chemotherapy; b after chemotherapy 

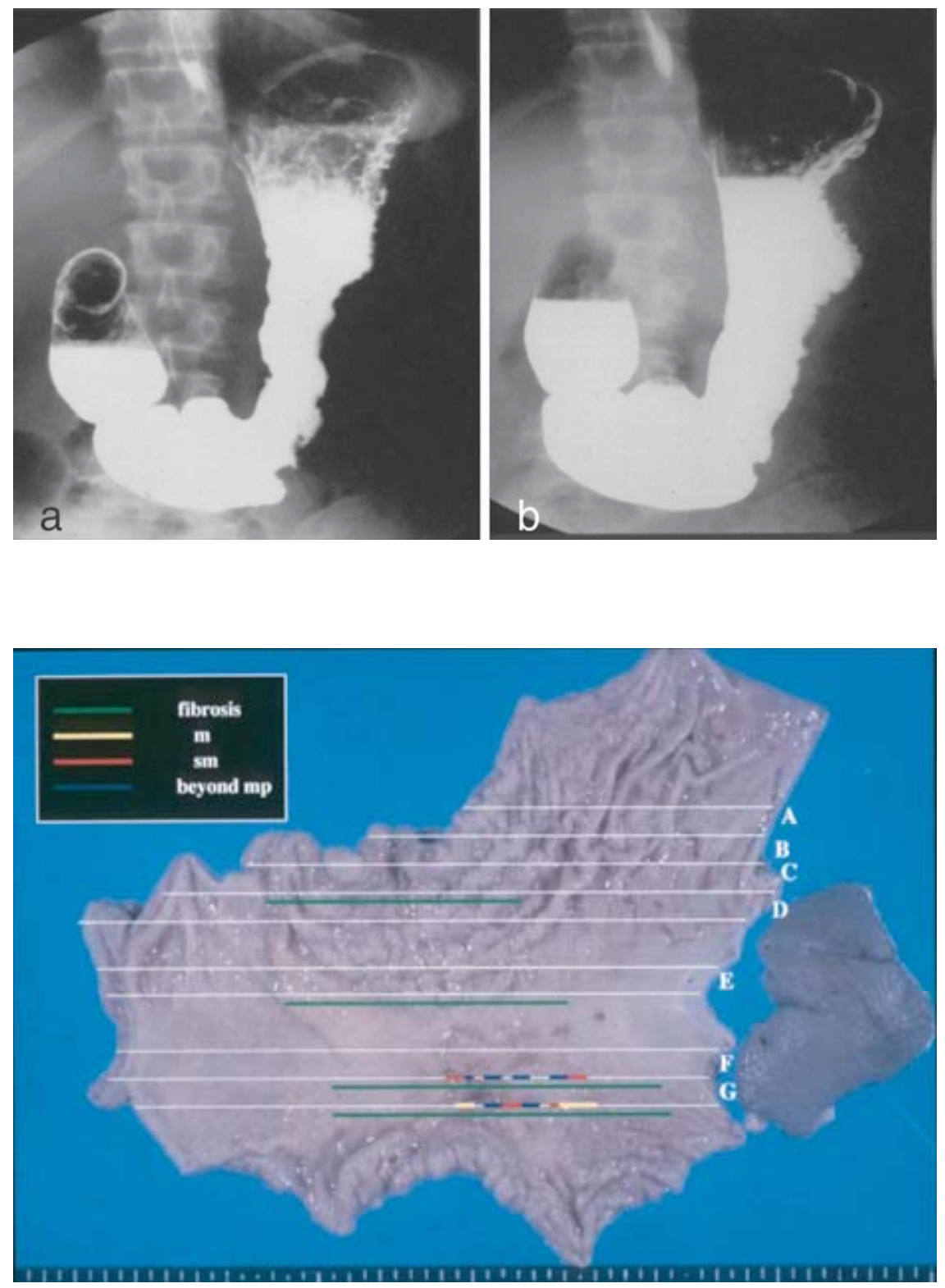

Fig. 2a,b. Fluoroscopic examination of case 4. a Before chemotherapy; b after chemotherapy
Fig. 3. Mapping of the cancerous lesion in the resected specimen of case 4. Green, Fibrosis; yellow, cancerous lesion limited to the mucosa $(\mathrm{m})$; red, cancerous lesion in the submucosa ( $\mathrm{sm})$; blue, cancerous lesion in the subserosa (beyond the muscularis propria [mp]. $A-G$, cut line of the specimen for histological examination
Pathological examination revealed a marked reduction in the distribution of cancer cells the patients with PR. Figure 3 shows the distribution of viable cancer cells in the resected specimen of case 4 . In case 3 , not only a marked shrinkage of the primary cancer in the stomach but also pathological findings suggestive of the possibility of disappearance of the cancer cells in the perigastric and paraaortic lymph nodes were noted (Fig. 4). Even though the depth of invasion of the primary tumor was T3 in the laparoscopic examination before treatment, the final depth in the pathological report was subserosal (T2). In this patient, the final stage of disease was Ib (T2N0) whereas the pretreatment stage of disease was IVa (T3N3). Marked downstaging was achieved by preoperative chemotherapy.
The two patients with noncurative resection died of the disease, whereas the three patients with curative resection are still alive without recurrence at 24,31 , and 33 months after initiation of the neoadjuvant chemotherapy.

\section{Discussion}

Treatment results for scirrhous gastric cancer, which is called diffuse gastric cancer, linitis plastica, or Borrmann type 4 tumor in the West, remain poor because of its aggressive biological behavior, even with recent advances in the field of chemotherapy and extended surgery. At our institution, because of the 

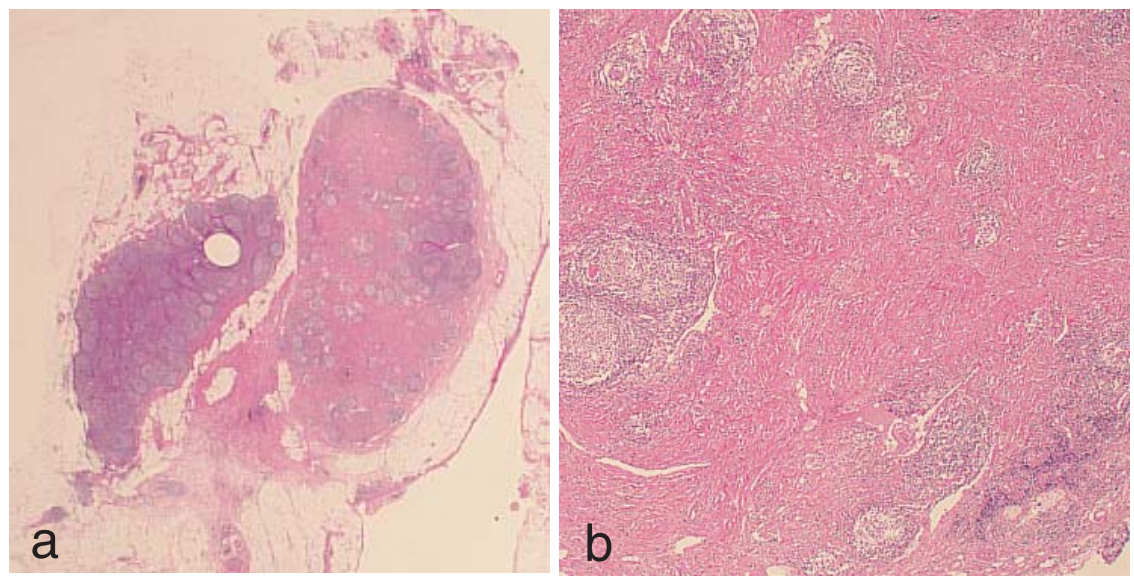

Fig. 4a,b. Hyalinized nodular lesions in lymph nodes of case 4 . These findings were seen in 10 of the 104 lymph nodes dissected. (No. 3, 6/9; No. 4d, 1/12; No. 8a,p, 2/9; No. 16b1, 1/9). a H\&E, $\times 10$; b $\mathrm{H} \& \mathrm{E}, \times 100$

frustration of being unable to improve survival, neoadjuvant chemotherapy using FAMTX was employed for patients with potentially resectable scirrhous gastric cancer in a phase II trial. The neoadjuvant chemotherapy seemed feasible, with a higher resectability rate, without any increase in the morbidity rate, compared with that of the historical controls. However, an analysis of 2-year survival when 20 patients were enrolled in the study showed no improvement over that of the historical controls. Myelosuppression was the major toxicity of the FAMTX regimen, and was observed as grade 3 or 4 neutropenia in 14 of the 20 patients $(70 \%)$. Eleven of these 14 patients required granulocyte colonystimulating factor (G-CSF) support. The overall response rate was $15 \%$ ( 3 PRs in 20 patients). Eighteen resected specimens showed only marginal histological effects (grade 0-Ib). For these reasons we stopped the trial [2].

There have been several reports concerning neoadjuvant chemotherapy for scirrhous gastric cancer [710]. Suga et al. [10] described the results of neoadjuvant chemotherapy using uracil and tegafur plus cisplatin for potentially unresectable scirrhous gastric cancer patients. Ascites in 8 of 13 patients (62\%) disappeared, and 10 of 28 patients became resectable [10]. However, all these studies were small in size and usually did not determine the survival benefits. The efficacy of neoadjuvant chemotherapy for scirrhous gastric cancer remains to be established because of the lack of good phase II and III studies.

As mentioned in the "Introduction", S-1 is an attractive oral anticancer agent with a high response rate and low toxicity. For scirrhous gastric cancer, S-1 showed a $33 \%$ response rate. The possibility of the outpatient use of S-1 also makes it attractive as a neoadjuvant chemotherapeutic agent. These are the reasons why we employed S-1 as an agent for neoadjuvant chemotherapy for scirrhous gastric cancer.
Peritoneal dissemination is the most frequent incurable factor at laparotomy. It is the most frequent type of recurrence after curative resection and is very difficult to detect in its early stage, except by laparoscopic examination.

Because we still have no promising regimen for peritoneal dissemination and because evaluation of the effect of chemotherapy against peritoneal dissemination is difficult, patients with visible peritoneal dissemination were evaluated by laparoscopic examination and excluded from this pilot study.

As shown in the "Results," a high response rate was obtained without high-grade toxicity. The rate of curative resection was $60 \%$, which is nearly the same as that with the FAMTX regimen. Histological effects of the neoadjuvant chemotherapy showed an unexpectedly high response by the primary lesions and the metastatic lymph nodes. We expected a change in the cytological examination results from positive to negative by the neoadjuvant chemotherapy, but two patients still showed positive results on cytological examinations after two courses of the neoadjuvant chemotherapy.

Even though the effects against peritoneal dissemination were questionable, a good impression concerning this neoadjuvant chemotherapy led us to conduct a phase II study of neoadjuvant chemotherapy using S-1 for scirrhous gastric cancer to confirm its feasibility and efficacy in a Japan Clinical Oncology Group (JCOG) trial.

\section{References}

1. Furukawa H, Hiratsuka M, Iwanaga T, Imaoka S, Ishikawa O. Extended surgery - left upper abdominal exenteration plus Appleby's method - for type 4 gastric carcinoma. Ann Surg Oncol 1997;4:209-14.

2. Takahashi S, Kinoshita T, Konishi M, Nakagohri T, Inoue K, Ono $\mathrm{M}$, et al. Phase II study of sequential high-dose methotrexate and 
fluorouracil combined with doxorubicin as a neoadjuvant chemotherapy for scirrhous gastric cancer. Gastric Cancer 2001;4;192-7.

3. Sugimachi K, Maehara Y, Horikoshi N, Shimada Y, Sakata Y, Miyachi Y, et al. An early phase II study of oral TS-1, a newly developed 5-fluorouracil derivative for advanced and recurrent gastrointestinal cancers. Oncology 1999;57:202-10.

4. Sakata Y, Ohtsu A, Horikoshi N, Sugimachi K, Mitachi Y, Taguchi T. Late phase II study of novel oral fluoropyrimidine anticancer drug TS-1 (1 M tegafur- $04 \mathrm{M}$ gimestat- $1 \mathrm{M}$ otastat potassium) in advanced gastric cancer patients. Eur J Cancer 1998;34:1715-20.

5. Koizumi W, Kurihara M, Nakano S, Hasegawa K. Phase II study of TS-1, a novel oral derivative of 5-fluorouracil, in advanced gastric cancer. Oncology 2000;58:191-7.

6. Japanese Gastric Cancer Association. Japanese classification of gastric carcinoma, 2nd English edition. Gastric Cancer 1998;1:10 24.
7. Mai M, Ogino T, Ueda H, Ooi A, Takahashi Y, Sawaguchi K, et al. Study on neoadjuvant chemotherapy of Borrmann 4 type carcinoma of the stomach and its clinical significance. Nippon Gan Chiryo Gakkai Shi (J Jpn Soc Cancer Ther) 1990;25:58697.

8. Maeda $\mathrm{O}$, Iwase $\mathrm{H}$, Mamiya $\mathrm{N}$, Nakamura M, Mizuno T, Nishio $\mathrm{Y}$, et al. A case of scirrhous cancer of the stomach which survived for more than 5 years after neoadjuvant chemotherapy with UFT (uracil and tegafur) and cisplatin. Intern Med 2000;39:239-44.

9. Eriguchi M, Osada I, Fujii Y, Takeda Y, Yoshizaki I, Akiyama N, et al. Pilot study for preoperative administration of 1-OHP to patients with advanced scirrhous type gastric cancer. Biomed Pharmacother 1997;51:217-20.

10. Suga S, Iwase H, Shimada M, Nishio Y, Ichihara T, Ichihara S, et al. Neoadjuvant chemotherapy in scirrhous cancer of the stomach using uracil, tegafur and cisplatin. Intern Med 1996;35:9306 . 